\title{
Turno de corta y posibilidad de los bosques de lenga (Nothofagus pumilio) en Tierra del Fuego (Argentina)
}

\author{
Rotation length and sustainable use of lenga (Nothofagus pumilio) forests in \\ Tierra del Fuego (Argentina)
}

\author{
GUILLERMO MARTINEZ PASTUR ${ }^{1}$, MARIA VANESSA LENCINAS ${ }^{1}$, RICARDO VUKASOVIC ${ }^{2}$, \\ PABLO PERI ${ }^{3,},{ }^{\text {BORIS DIAZ }}{ }^{3}$, JUAN MANUEL CELLINI ${ }^{5}$ \\ ${ }^{1}$ Centro Austral de Investigaciones Científicas. cc 92 (9410) Ushuaia, Tierra del Fuego, Argentina. \\ ${ }^{2}$ Consultora "Servicios Forestales", Río Grande, Tierra del Fuego, Argentina. \\ ${ }^{3}$ Universidad Nacional de la Patagonia Austral, Río Gallegos, Santa Cruz, Argentina. \\ ${ }^{4}$ Instituto Nacional de Tecnología Agropecuaria, Río Gallegos, Santa Cruz, Argentina. \\ ${ }^{5}$ Universidad Nacional de La Plata. La Plata, Buenos Aires, Argentina. \\ E-mails: cadicforestal@arnet.com.ar; cadicforestal@gmx.net
}

\begin{abstract}
SUMMARY
Forest regional planning is an important tool for developing forest policies for the protection of natural forests, the use of sustainable management and multiple use principles. It is necessary to use parameters and reliable models that simulate forest production and alternative land use capabilities. The forest inventory is the primary tool, but without precise estimations of rotation length it is not possible to obtain reliable data of sustainable forest use. For these reasons, the objective was to define a simple methodology to calculate the rotation length (harvest diameter of $40 \mathrm{~cm}$ ), by analyzing the interaction and influence of: (a) livestock browsing pressure, (b) site quality, and (c) silvicultural management. Sustainable use of Tierra del Fuego forests in Argentina was investigated using several alternatives. Some assumptions were adopted for the silvicultural system, forest management objectives, stand growths and browsing pressure. These were methodologies and biometrical models selected from the bibliography. The methodology for rotation length estimation was defined and composed of three phases of tree growth, the establishment stage, initial growth and diameter growth. This was undertaken while considering livestock, silvicultural management and site quality. Models for rotation length estimation are presented and the effects of livestock pressure and silviculture (with or without) were analyzed in forests that varied between 94 and 200 years of age. Sustainable forest use calculated for the productive forests varied from $913 \mathrm{ha} /$ year for the current situation to 1692 ha/year with silviculture and without browsing pressure. The rotation length varied considerably according to the site quality and the type of management used for the forest. The application of a single standard rotation length in all forest situations introduced large errors, with the weighted average data obtained being higher than those suggested for lenga. Finally, according to the rotation length values and sustainable forest use of Tierra del Fuego forests, several alternatives are discussed for adjusting harvesting rates toward a sustainable management of the forest resource.
\end{abstract}

Key words: forest planning, forest management, sustainability, silviculture, browsing.

\section{RESUMEN}

La ordenación forestal regional es una herramienta indispensable para desarrollar políticas forestales que protejan los bosques naturales, en el marco del manejo sustentable y del uso múltiple. Para ello se debe contar con parámetros y modelos confiables que simulen alternativas de producción y de uso del suelo. El inventario forestal es la herramienta base, pero que sin cálculos precisos de turno forestal no puede brindar datos confiables de posibilidad. Por lo que el objetivo del presente trabajo fue definir una metodología sencilla para calcular el turno forestal de los bosques de Tierra del Fuego (para un diámetro de cosecha de $40 \mathrm{~cm}$ ), analizando la interacción e influencia de: (a) la presión del ganado, (b) la calidad de sitio, y (c) el manejo silvícola; y definir la posibilidad del 
BOSQUE 25(1): 29-42, 2004

Turno de corta y posibilidad de los bosques de lenga (Nothofagus pumilio) en Tierra del Fuego (Argentina)

bosque de Tierra del Fuego en Argentina bajo diferentes alternativas. Se definieron supuestos de trabajo para el sistema silvícola empleado, objetivos del manejo forestal, crecimientos y presión del ganado. Las metodologías y modelos biométricos se seleccionaron de la bibliografía. A partir de los supuestos y los modelos se definió una metodología para el cálculo del turno forestal basada en la disgregación en tres fases del crecimiento (etapa de establecimiento, de crecimiento inicial y de crecimiento en diámetro) y considerando la influencia del ganado, el manejo silvícola y la calidad de sitio. En este trabajo se presentan los modelos desarrollados para el cálculo del turno forestal actual, con o sin presión del ganado y con o sin manejo silvícola, que varían entre 94 y 200 años; así como la posibilidad obtenida para los bosques productivos que va desde 913 ha/año para la situación actual hasta 1692 ha/año para la situación potencial (con silvicultura intensiva y sin ganadería). El turno forestal varía considerablemente de acuerdo a la calidad de sitio y al manejo. No es lógico aplicar un valor fijo de turno forestal a la totalidad de los bosques bajo manejo, siendo los promedios ponderados obtenidos superiores a los sugeridos en la bibliografía para la lenga. Finalmente, y de acuerdo a los valores de turno forestal obtenidos y la posibilidad de los bosques de Tierra del Fuego, se discuten alternativas para adecuar las tasas de aprovechamiento a un manejo sostenible del recurso.

Palabras claves: planificación forestal, manejo forestal, sustentabilidad, silvicultura, ganado.

\section{INTRODUCCION}

Los componentes dominantes de los bosques de Tierra del Fuego corresponden a especies del género Nothofagus Blume. Poseen una sucesión simple, predecible y de alta resiliencia (gran amplitud, baja maleabilidad e histéresis) (1). La dinámica natural incluye hechos catastróficos (incendios, avalanchas o volteos de viento), regenerando en bosquetes coetáneos. Al regenerar los bosques, sus renovales crecen en masas compactas compitiendo por la luz, siendo el principal factor que afecta el desarrollo de las plantas ya que influye sobre el metabolismo de las mismas (2). La investigación forestal ha permitido el desarrollo de varios modelos silvícolas en numerosas especies productivas, siendo Nothofagus pumilio (Poepp. et Endl.) Krasser (lenga) la que más se adecua a las demandas del manejo forestal del bosque nativo. Los bosques son factibles de ser manejados bajo diferentes regímenes silvícolas, aprovechando su madera y regenerándoselos por diferentes vías: cortas de entresaca (por floreo o cortas selectivas), talas rasas (en fajas o en bosquetes) o cortas de protección (sucesivas o por única vez) (3-8). Los métodos más difundidos apuntan a la transformación del bosque natural en un sistema regular (o sea, de bosques primarios a bosques de segundo crecimiento - principalmente fustales). Si bien esto no ha ocurrido en muchos rodales aprovechados por floreo, sí puede observarse en amplias zonas donde se han practicado cortas más intensivas. Por otra parte, la calidad de sitio influye sobre el crecimiento $(9,10)$, debiendo incluirse este factor al analizar cualquier variable que sea directa o indirectamente afectada por el mismo.

Existen diferentes mercados para la madera de lenga, requiriendo cada industria una exigencia determinada para un tipo de producto en particular. En Tierra del Fuego (Argentina) predominan los aserraderos medianos que requieren madera de alta calidad para el aserrado, destinada a la producción de madera de medianas y grandes escuadrías. En muchos casos, estas exigencias limitan las dimensiones y calidades de trozas que pueden absorber (11).

Numerosas investigaciones demuestran que los Nothofagus no pueden regenerarse cuando son sometidos a disturbios sistemáticos y/o permanentes (12), siendo extremadamente vulnerables al sobrepastoreo (ganado ovino, equino y vacuno, cérvidos, conejos y guanacos) (13-21). En Tierra del Fuego (Argentina), una parte de los bosques es fuertemente degradada por el sobrepastoreo vacuno, principalmente en los campos destinados para las veranadas, así como por ramoneo del guanaco (Lama guanicoe Muller), especie nativa que usualmente incorpora al Nothofagus en su dieta a lo largo de todo el año $(15,22)$. Es por ello que la gran incompatibilidad del manejo forestal en Tierra del Fuego es la actividad ganadera. El ganado dentro del bosque complementa su dieta con brotes tiernos de renovales de árboles. A consecuencia de ser destruido el ápice, brotan las yemas laterales debido a la pérdida de la dominancia apical del mismo. El renoval pierde, así, el porte arbóreo, tomando una forma arbustiva. En la gran mayoría de los casos termina por morir, pero si 
BOSQUE 25(1): 29-42, 2004

Turno de corta y posibilidad de los bosques de lenga (Nothofagus pumilio) en Tierra del Fuego (Argentina)

escapa a la presión del ganado crecerá con un porte arbóreo defectuoso y muchas de sus cualidades económicas se perderán. Es posible encontrar extensos manchones con individuos que no superan los $50 \mathrm{~cm}$ de altura, intensamente ramoneados que nunca alcanzarán un estado adulto normal. También se pueden observar árboles que escaparon a la presión del ganado, desarrollando un fuste por encima de una altura de ramoneo, que se ubican en los lugares más protegidos o en las zonas centrales de los manchones en regeneración. Por otra parte, se pueden observar lugares donde el bosque logró escapar a la presión del ganado, pero donde la mayoría de los individuos han crecido con mala forma forestal: fustes retorcidos, bifurcados o con muy mala sanidad por las heridas recibidas.

Las acciones que impone un plan de manejo (aplicación y/o remoción de presiones) deben considerar a los factores de tensión naturales a los que el bosque está preadaptado y que ya tiene respuestas, para alcanzar el nivel deseado de estructura y función (1). El desafío de una política forestal se podría simplificar en cómo proteger los bosques naturales y permitir que continúen satisfaciendo las necesidades humanas, y la solución pudiera estar en el manejo sustentable y de uso múltiple (12). Muchas veces los beneficios económicos y sociales hacen que la erradicación de estas especies introducidas sea impracticable por razones políticas (18). Por esta razón, y considerando que el ganado es una de las actividades agropecuarias más importantes en la Isla Grande de Tierra del Fuego (Argentina), se consideró importante incluirlo en este análisis, para evaluar los efectos sobre el turno forestal, y dar las pautas de alternativas de manejo que tiendan a minimizar los efectos de estos animales sobre la futura posibilidad del bosque fueguino. El objetivo del presente trabajo fue definir una metodología sencilla para calcular el turno forestal de los bosques de $N$. pumilio, a través de los años, que son necesarios para que los árboles dominantes de un rodal de un bosque secundario alcancen un diámetro de $40 \mathrm{~cm}$, analizando la interacción e influencia de: (a) la presión del ganado, (b) la calidad de sitio y (c) el manejo silvícola. Así como para definir la posibilidad del bosque de Tierra del Fuego (Argentina) bajo diferentes alternativas de política y manejo forestal. Este trabajo brinda una metodología sencilla para complementar la información generada por un In- ventario Forestal Regional que apunte al desarrollo de un Plan de Ordenación Territorial.

\section{MATERIAL Y METODOS}

Metodología del cálculo del turno: Como primera medida, se definió, dentro de los objetivos del manejo forestal, un diámetro deseable de extracción (diámetro objetivo del aprovechamiento forestal) basado en la industria forestal que actualmente existe en Tierra del Fuego (Argentina) (11, 23). Se definió como el árbol deseable a aquel que tiene un diámetro promedio de $40 \mathrm{~cm}$ al DAP (diámetro normal a $1,3 \mathrm{~m}$ ). Por otra parte, se trabajó con el supuesto de que los bosques, que se manejen a futuro, serán mayormente regulares y se aprovecharán dentro de la fase de crecimiento óptimo final (6). Este supuesto se basa en la regeneración de los bosques bajo un sistema de cortas de protección. Esto no ocurre en la actualidad, ya que no se realizan las cortas finales, pero es posible que ocurra en corto plazo debido a la escasez de materia prima, así como la posible instalación de nuevas industrias de síntesis que pueden absorber materia prima de peor calidad. Por otra parte, existe una tendencia por parte de los aserraderos a adecuarse tecnológicamente a la materia prima, pudiendo absorber un mayor porcentaje de la madera cosechada del bosque, aumentando sus rendimientos y disminuyendo sus costos.

Para el cálculo del turno se consideraron tres fases:

- Etapa de establecimiento, que es la etapa en que la regeneración se instala y reúne el vigor (biomasa de follaje mínima) suficiente para comenzar un desarrollo de crecimiento exponencial;

- Etapa de crecimiento inicial, que es el período en que los nuevos árboles forman masas cerradas hasta lograr una altura de 1,3 m, siendo el período de mayor vulnerabilidad al ramoneo del ganado; y

- Etapa de crecimiento en diámetro, donde los individuos crecen hasta alcanzar el diámetro final deseado. Las dos últimas etapas son afectadas por los factores estudiados (calidad de sitio, ganado y silvicultura), por lo que se simularán diferentes alternativas. 
BOSQUE 25(1): 29-42, 2004

Turno de corta y posibilidad de los bosques de lenga (Nothofagus pumilio) en Tierra del Fuego (Argentina)

Cálculo del turno natural: Se trabajó con datos de crecimiento provenientes de diez lugares de Tierra del Fuego y Santa Cruz (Argentina) (24) y de la base de datos utilizada para la construcción de ecuaciones de crecimiento de lenga $(25,26)$. Se utilizaron mediciones de crecimiento periódico cada cinco años, de un total de 116 árboles dominantes a lo largo del rango de calidades de sitio planteadas en Martínez Pastur et al. (10). Los árboles muestreados estaban creciendo en masas puras de densidad completa. El modelo ajustado fue el de Chapman-Richards, presentado por Mitscherlich (27) y posteriormente por Richards (28).

$$
\text { DAP }=a(6-C S) b\left(1-e^{c E^{d(6-C S)^{f}}}\right)
$$

donde DAP es diámetro normal a 1,3 m $(\mathrm{cm}), \mathrm{CS}$ es la clase de sitio donde 1 es la más alta y 5 la más baja de acuerdo a Martínez Pastur et al. (10), E es la edad (años) y abcdf los parámetros ajustados.

Esta ecuación se caracteriza por su flexibilidad, exactitud y cualidades biológicas (29). El ajuste se realizó mediante técnicas de regresión no lineal, y se evaluó mediante el coeficiente de determinación $\left(\mathrm{r}^{2}\right)$, el análisis de los residuales (ANARE), el error estándar residual (EER) y el error absoluto medio (EAM).

Los crecimientos se modelaron hasta alcanzar el diámetro objetivo de $40 \mathrm{~cm}$. A partir de este modelo se obtuvieron los años necesarios para la etapa de crecimiento en diámetro. Con las edades necesarias para alcanzar el diámetro deseado y las calidades de sitio se realizó una regresión lineal simple para obtener un valor estandarizado a lo largo de las diferentes clases de sitio.

Este mismo período se utilizó para los bosques con presión ganadera, por considerar que los árboles que se encuentran por arriba del DAP estarían fuera de peligro de ramoneo. Estas consideraciones incluyen el supuesto de que un árbol (que fue ramoneado o no) al llegar al 1,3 m crecerá de la misma forma hasta alcanzar los $40 \mathrm{~cm}$ de DAP. Cabe destacar que este último punto también fue tenido en cuenta cuando se realizaron otros trabajos (10), encontrando una alta variabilidad en el crecimiento en altura desde el tocón hasta el DAP, pero una alta homogeneidad desde el 1,3 m hasta la altura total de los individuos. Los cálculos para la etapa de establecimiento y la etapa de crecimiento inicial se realizaron a base de los datos antes mencionados (10) y a estudios de establecimiento y desarrollo de regeneración de lenga en Tierra del Fuego $(21,30)$.

Cálculo del turno con ganado: En este estudio se consideró que el ganado afecta negativamente la segunda etapa analizada (crecimiento inicial), impidiendo el desarrollo normal de los renovales durante un mayor período de tiempo, siendo más fuerte la presión a medida que empeora la calidad de sitio. Una vez establecida la regeneración, se consideró que la misma tardará el triple de tiempo en alcanzar el 1,3 m de altura que un rodal sin presión de ganado. Este valor se consideró como un valor promedio de las situaciones que ocurren en Tierra del Fuego, ya que la mima es variable de acuerdo a la carga animal que existe en el predio y su historia. Por otra parte, para el cálculo de la posibilidad se consideró un descuento de superficie productiva cuando el bosque posee presión ganadera.

Cálculo del turno silvícola: Se realizaron simulaciones de acuerdo a datos de diez árboles dominantes bajo crecimiento libre. Se partió del supuesto de que esos árboles presentan un comportamiento similar a aquellos individuos a los que se les aplican tratamientos silviculturales y que se ven favorecidos en su desarrollo. Luego, y de la misma forma que para el cálculo del turno natural, con las edades y los sitios, se realizó una regresión linear simple, de modo de obtener un valor estandarizado a lo largo de las diferentes clases de sitio. De este modo se definieron los años que son necesarios para completar la etapa de crecimiento en diámetro. Se partió del supuesto de que no existe ganadería dentro de los rodales bajo manejo, basados en los principios desarrollados en la introducción de este trabajo.

Porcentajes de afectación de clases de sitio y presión ganadera: A partir de los datos presentados en el Inventario Forestal de la provincia de Tierra del Fuego (Argentina) (23), se extrajeron los porcentajes de calidades de sitio que existen en los bosques de la Isla Grande, discriminados de acuerdo a la presión ganadera que se detectó en los mismos. Dichos porcentajes fueron utilizados para la ponderación de los turnos parciales (para cada clase de sitio, con y sin silvicultura, con y sin presión del ganado) a los fines de obtener un turno promedio para las distintas modelizaciones. 
Cálculo de la posibilidad: En este trabajo la posibilidad se define como el producto maderero que se puede extraer de un bosque, de modo que se mantenga una tasa de extracción uniforme del mismo a lo largo del tiempo. La forma más adecuada es realizarla por medio de un volumen de aprovechamiento, considerando el crecimiento de los rodales. Sin embargo, su realización requiere de un conocimiento muy detallado del recurso, por lo que no ha demostrado ser una herramienta útil para la planificación regional de países en desarrollo. Una forma más sencilla es considerar la posibilidad de acuerdo a la superficie afectada por año, siendo la forma seleccionada para este estudio. El principal justificativo para utilizar superficies se basa en que diferentes industrias obtienen diferentes rendimientos para una misma superficie de bosque. Por ejemplo, hay aserraderos que obtienen un rendimiento de trozas por hectárea de entre $130-150 \mathrm{~m}^{3}$ para sitios de calidad II-III (7), mientras que otros aprovechan menos de $50 \mathrm{~m}^{3} / \mathrm{ha}$ para las mismas calidades, dejando abandonadas en el monte entre $20-50 \mathrm{~m}^{3}$ de trozas por hectárea, quedando el resto en pie. Si se considera la posibilidad como volúmenes de aprovechamiento, éstos arrojarán diferentes superficies para diferentes aserraderos. Al considerar la posibilidad por superficie, es posible obtener una tasa uniforme que puede rendir diferentes volúmenes de acuerdo a las diferentes industrias que los utilicen para obtener su materia prima. Es por ello que independientemente del volumen aprovechado, la superficie de la posibilidad debería ser la misma para todos los años. Un punto a tener en cuenta cuando hablamos de posibilidad, es que la superficie a la que se hace referencia es aquella que año tras año es incorporada al manejo forestal. Sin embargo, en la medida en que los tratamientos intermedios prescritos se vayan realizando la superficie intervenida anualmente podría ser mayor, mientras que en un sector se realizarán cortas de protección, en otros se irán realizando raleos o cortas preparatorias.

La superficie de bosque productivo de Tierra del Fuego (Argentina) se obtuvo a partir del análisis de imágenes satelitales (año 1995) y de la base de datos del S.I.G. que posee la Subsecretaría de Recursos Naturales de la Provincia de Tierra del Fuego (Argentina) (31). A la superficie de bosque productivo se le realizaron descuentos de superficie (por protección, caminos, canchones y fragmentación del bosque) que son los que generalmente se producen durante el aprovechamiento y por un uso ganadero del bosque. Los tres primeros datos surgen de valores estimativos provenientes de estudios de planes de manejo presentados por aserraderos locales $(32,33)$. El valor de degradación por ganado surge como un valor conservador de observaciones de campo. Como ejemplos de tales observaciones se pueden citar: (1) la falta de recuperación debida al sobrepastoreo en los sectores de aprovechamiento del Lago Yehuin - Lago Chepelmut (Argentina); (2) la escasa instalación de regeneración e imposibilidad de crecimiento posterior en el sector del incendio de 1978 en las márgenes del Lago Fagnano (Argentina) debidas a la alta tasa de ganado que pastorea en la zona; y (3) la malformación de los individuos que escaparon al ramoneo y la alta heterogeneidad de los rodales resultantes de las talas rasas practicadas en el Cuartel Forestal Río Valdez (Argentina), debidas a la gran cantidad de ganado introducido por establecimientos no autorizados.

\section{RESULTADOS Y DISCUSION}

Turno potencial: Para el cálculo del turno potencial (sin silvicultura y sin presión del ganado) se consideró el crecimiento natural de los árboles dominantes de bosques de segundo crecimiento, creciendo en masas regulares compactas, donde el autorraleo natural (34) limita el número de individuos que van quedando en el rodal. La supervivencia se produce de acuerdo a la dominancia de los individuos: los dominantes y codominantes sobrevivirán, mientras que los del dosel inferior (intermedios y suprimidos) son los que generalmente morirán. En este sentido, considerar el crecimiento de los árboles dominantes nos arrojará un valor adecuado para el cálculo del turno. Por otra parte, al trabajar en bosques de densidad completa, se consideró que la influencia de la densidad no es significativa, que se pone de manifiesto en la homogeneidad obtenida dentro de las muestras de un mismo sitio de muestreo. El crecimiento se modelizó a la altura del DAP, discriminando de acuerdo a la calidad de sitio y hasta un diámetro comercial de $40 \mathrm{~cm}$ (figura 1), obteniendo los siguientes estadísticos (cuadro 1). Como es previsible, a medida que el sitio empeora (de clases de sitio altas I y II, hasta la peor clase en sitio V) los 
BOSQUE 25(1): 29-42, 2004

Turno de corta y posibilidad de los bosques de lenga (Nothofagus pumilio) en Tierra del Fuego (Argentina)

\section{CUADRO 1}

Coeficientes y estadísticos del modelo de crecimiento diamétrico.

Parameters and statistic of the diameter growth model.

\begin{tabular}{|ll|}
\hline Coeficientes ajustados \\
$\mathrm{a}=-51,4571$ \\
$\mathrm{~b}=\quad 1,2868$ & \\
$\mathrm{c}=\quad 0,0017$ & \\
$\mathrm{~d}=\quad-0,1828$ & \\
\hline & \\
$\mathrm{r}^{2}$ ajustado & 0,9502 \\
Error estándar de la estimación & 2,4588 \\
Error absoluto medio & 1,7119 \\
\hline
\end{tabular}

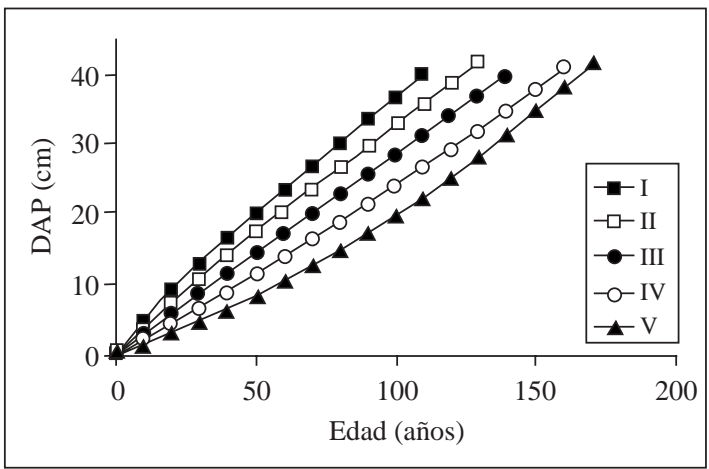

Figura 1. Crecimiento diamétrico de árboles dominantes en bosques de densidad completa sin intervención silvícola a lo largo de un gradiente de clases de sitio.

Diameter growth of dominant trees along a site class gradient in well stocked stands without silvicultural interventions.

árboles tardan más tiempo en alcanzar el diámetro deseado. Con los valores de edad obtenidos al alcanzar un diámetro de $40 \mathrm{~cm}$, y su correspondiente valor de calidad de sitio, se realizó una regresión lineal para estandarizar los valores, obteniendo el siguiente modelo y residuos (figura 2). El modelo quedó definido como:

$$
\operatorname{Edad}(\operatorname{años})=96,4+14,4 \text { CS }
$$

donde Edad representa los años para un DAP de $40 \mathrm{~cm}$ y CS es la clase de sitio (1 a 5) (10).

Para el cálculo del establecimiento de la regeneración se utilizaron los datos provenientes de un estudio de regeneración en la Estancia San Justo $(21,30)$ que se realizó en un rango de calidad de

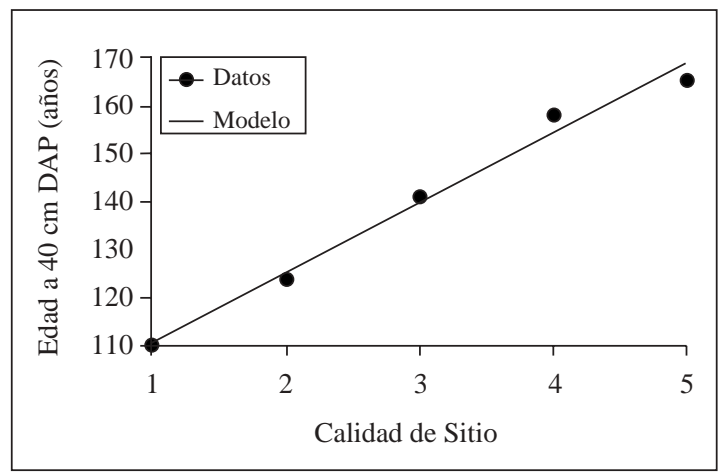

Figura 2. Tiempo necesario para alcanzar un diámetro comercial de $40 \mathrm{~cm}$ según la calidad de sitio del rodal (modelo y residuos).

Years required to reach a $40 \mathrm{~cm}$ commercial stem diameter according to the site quality of the stand (model and residuals).

sitio media alta (II-III), y donde un período de cinco años posterior al aprovechamiento fue suficiente para lograr la instalación de una abundante regeneración de 10-15 cm de altura. Estos datos son coincidentes a los obtenidos por Schmidt y Urzúa (6), Schmidt y Caldentey (35) y Schmidt et al. $(36,37)$. El valor de cinco años se estableció como una constante a lo largo de todos los sitios, aunque es posible que en sitios de peor calidad, los tiempos de instalación sean mayores. Ya que no existen trabajos que aporten datos al respecto, se decidió utilizar el valor con el que ya se contaba.

El crecimiento para llegar hasta los $1,3 \mathrm{~m}$ es sumamente variable, siendo uno de los motivos principales para eliminarlos de cualquier análisis de crecimiento en altura (10). El tiempo de instalación de las plántulas es el principal responsable de dicha variabilidad. En muchas ocasiones, la regeneración que vemos instalada proviene del banco de plántulas que el bosque posee junto al sotobosque, y que se renueva año a año $(21,30)$. Algunas plantas sobreviven por más de veinte años esperando que se abra el dosel de protección (por fenómenos naturales o por un aprovechamiento), mientras que otras se instalan en los años siguientes a la cosecha, aumentando con mucho vigor y rápido crecimiento. A ello se le suma, el fenómeno de las semillazones cíclicas que posee la lenga, donde hay años de alta escasez y años de gran producción de semillas. Este fenómeno tiene un ciclo aproximado de siete años (35), al igual que otros Nothofagus (38). Bajo estas consideraciones y con los datos provenientes de análisis fustales 
de la misma muestra antes citada, se obtuvieron los valores de crecimiento en altura desde el nivel del suelo hasta los 1,3 $\mathrm{m}$ a lo largo de un rango de calidades de sitio (figura 3). Como era previsible, sitios de mejor calidad arribaron en menor tiempo a dicha altura que sitios de peor calidad. En la figura 4 se presentan los turnos obtenidos para cada clase de sitio en un bosque natural. Podemos observar que un sitio de alta calidad (clase de sitio I) alcanza el diámetro buscado en un $65 \%$ del tiempo que requiere un sitio de clase V. Estos resultados resaltan la importancia del sitio en la planificación de la ordenación de un bosque de lenga.

Turno en la situación actual: Para el cálculo del turno en la situación actual se consideró la presencia de ganado de acuerdo al inventario forestal de Tierra del Fuego (23) y a la inexistencia de tratamientos silviculturales intermedios en los bosques. La presencia del ganado afecta las primeras etapas del crecimiento de los bosques de acuerdo a la carga animal, por lo que el análisis del ganado se simplificó enormemente, ya que son múltiples los factores que influyen sobre el bosque: (a) por un lado, hay que considerar las zonas donde se realiza la ganadería, ya que hay zonas de veranada exclusiva (centro de la Isla Grande de Tierra del Fuego) y lugares donde también se realiza invernada (costas del Lago Fagnano y Canal Beagle en Argentina), donde estos últimos recibirán mucho más presión que los otros; (b) la carga animal

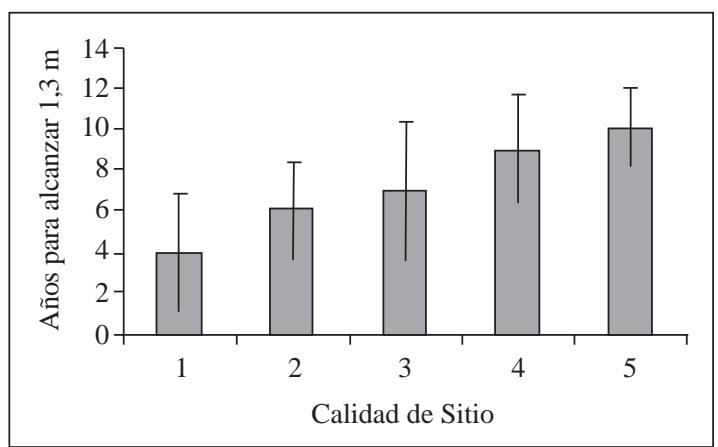

Figura 3. Años necesarios para alcanzar los $1,3 \mathrm{~m}$ de altura en bosques naturales sin presión del ganado a lo largo de un rango de clases de sitio (las barras representan el error estándar).

Years required to reach $1.3 \mathrm{~m}$ in height in a natural forest without browsing pressure along different site quality classes (bars represent the standard error).

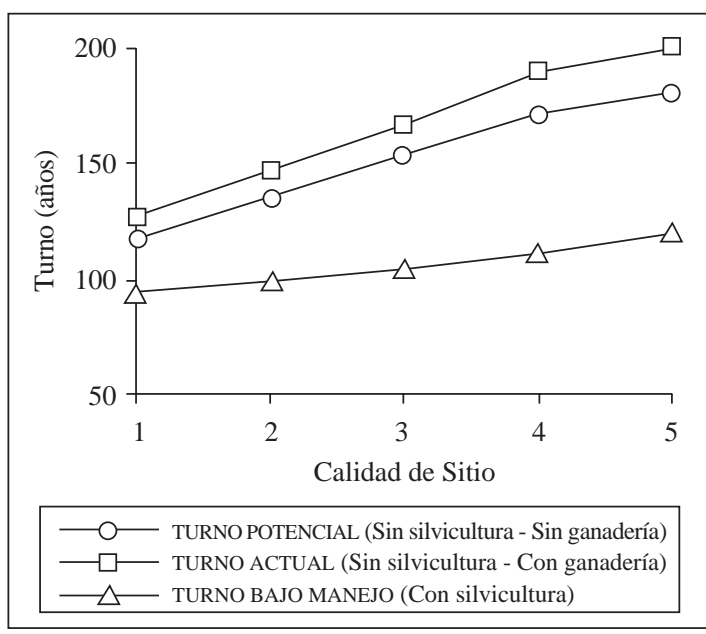

Figura 4. Turno forestal para bosques naturales, con y sin presión ganadera, con y sin manejo silvícola a lo largo de un gradiente de sitios.

Rotation lengths for natural forest with and without browsing pressure and silvicultural management along different site quality classes.

es un factor muy importante, estimada en un animal cada 5-10 ha, pero hay sectores donde se observan densidades mayores de animales (por alambrados o bordes de vegas), y (c) el tipo de ganado, ya que no es lo mismo el daño que producen las ovejas que los caballos o las vacas. Es posible observar que el daño que produce la oveja es más considerable que los otros dos y, a su vez, la vaca muchas veces voltea árboles jóvenes para poder comer su follaje (cuando no hay otro alimento disponible). A lo largo de la Patagonia, el ganado y los cérvidos son considerados como el principal factor de degradación del bosque y son ampliamente tratados en la bibliografía (16, 17, 18), pero no son tenidos en cuenta al elaborar políticas de desarrollo o de uso del suelo en la planificación regional de zonas boscosas. En las modelizaciones (figura 4) se puede observar que un sitio de calidad alta (clase de sitio I) ve retrasado el tiempo de producción en un $15 \%$ del tiempo respecto de un bosque sin ganado, mientras que un sitio de mala calidad (clase de sitio V) tardará un $24 \%$ más tiempo que el que necesitaba para alcanzar un diámetro deseable cuando crecía sin presencia de ganado. Por otra parte, la diferencia entre sitios se amplía hasta un $61 \%$, ya que sitios de mala calidad (clase de sitio V) son más afectados que sitios de buena calidad (clase de sitio I). 
Turno bajo manejo silvícola: Como ya fue planteado, para el cálculo del turno bajo manejo silvícola se consideró el incremento de árboles con crecimiento libre en bosques de segundo crecimiento o en bosques irregulares. El criterio para utilizar estos árboles se fundamenta en que si se encontraron esos crecimientos en la naturaleza, también serán posibles de ser obtenidos en bosques bajo manejo silvícola. Los resultados presentados en la bibliografía son muy alentadores (8), llegando hasta $20 \mathrm{~m}^{3} / \mathrm{ha}$ en bosques bajo manejo. Schmidt y Caldentey (35) citan crecimientos de entre 4 y $6 \mathrm{~m}^{3} / \mathrm{ha}$ para bosques raleados en clase de sito IV-V. Por otra parte, en raleos de $N$. betuloides (Mirb.) Oerst. de Tierra del Fuego en Argentina se obtuvieron crecimientos de hasta 17 $\mathrm{m}^{3} / \mathrm{ha}$ (39). Los crecimientos obtenidos a partir de árboles en crecimiento libre variaron entre 85 años (clase de sitio I) hasta 106 años (clase de sitio V). Si bien los árboles tardan más tiempo en alcanzar el diámetro deseado a medida que la calidad de sitio empeora, éstos lo realizan en un tiempo más corto que el planteado para bosques bajo crecimiento natural. Estos crecimientos fueron incorporados a los cálculos del turno con silvicultura, presentando los valores obtenidos en la figura 4. Podemos observar que a lo largo de todo el gradiente de sitios se produce una disminución del tiempo necesario para alcanzar el diámetro deseado. En sitios de alta calidad (clase de sitio I), dicho diámetro se alcanza en el $75 \%$ del tiempo necesario de un bosque natural sin manejo y en un $66 \%$ del tiempo necesario de un bosque natural con alta presión ganadera. Asimismo, se observa que en sitios de mala calidad (clases de sitio V), el diámetro comercial se alcanza en un $64 \%$ del tiempo, mientras que en un bosque con ganadería se alcanza en un $52 \%$ del tiempo que dura el turno.

Posibilidad del bosque productivo de Tierra del Fuego: Contar con un valor de posibilidad para un bosque sometido a actividades productivas, permite definir las tasas de corta permisibles para lograr un manejo sostenible del recurso. Cuando se trata de grandes superficies boscosas, como las existentes en la provincia de Tierra del Fuego (Argentina) y debido a que es una planificación a largo plazo, sufre de los problemas propios de este tipo de proyectos que puede definirse como: "Grandes Proyectos, Grandes Problemas" (40). Al calcular la posibilidad se tienen en cuenta parámetros que deberán ser actualizados en forma continua y sin interrupción; por ejemplo, al decidirse la creación de una reserva turística, o un nuevo polo productivo, o la cesión de tierras del Estado al dominio privado, se van modificando las superficies productivas de bosque al modificarse el uso a las que el Estado o sus dueños destinan a las mismas. Estas modificaciones afectan la posibilidad del bosque bajo análisis.

Se analizaron distintas alternativas de cálculo de la posibilidad, para discutir la influencia de la ganadería y la silvicultura dentro del marco de la situación actual de los bosques de Tierra del Fuego (Argentina) y el impacto que tendría la implementación de determinadas políticas forestales en el sector productivo en un mediano y largo plazo.

a) Considerando la situación actual. En la actualidad en los bosques de Tierra del Fuego (Argentina) no se aplican las prescripciones silvícolas que se proponen en las planificaciones que se presentan ante la Autoridad de Aplicación, por ejemplo, Fitzgerald (32) y Frem (33). El sistema de regeneración propuesto para estos bosques es el de cortas de protección (6). Sin embargo, en la gran mayoría de los casos se realiza una corta selectiva fuerte, donde quedan muchos sectores poco aprovechados que con el tiempo cerrarán su canopeo, sin llegar a regenerar al rodal en forma completa. Por otra parte, cuando la calidad de sitio del bosque es buena, se aplica correctamente la corta inicial de la corta de protección, pero nunca se llevó a cabo la corta final propuesta en el tratamiento silvícola. Eso implica que el tratamiento silvícola propuesto es una corta de regeneración con retención dispersa (41). Por otra parte, existen varios establecimientos que realizan ganadería en bosques con aprovechamiento forestal. Este pastoreo causa grandes daños en las plántulas que crecen en los bosques bajo regeneración.

En el cuadro 2 se presentan los cálculos para la posibilidad en la situación actual en los bosques fueguinos (con presión ganadera y sin manejo silvícola posterior a la corta de regeneración). Los bosques de producción (213.715 ha) incluyen los bosques naturales, con y sin intervención, fiscales y privados que se encuentran fuera del Parque Nacional en todo el ámbito de la provincia de Tierra del Fuego (Argentina) independientemente de su accesibilidad (31). Los descuentos para el cálculo de las superficies efectivas se realizaron 
considerando la ausencia de ganado $(15 \%)$ o la presencia de ganado (45\%). La presencia de ganado implica la degradación parcial del bosque, tanto evitando la regeneración del mismo como la pérdida de valor económico de los árboles regenerados (bases retorcidas, troncos bifurcados, etc.). El turno forestal promedio se calculó a base de un promedio ponderado (de acuerdo a la superficie de bosque de determinada calidad de sitio y su correspondiente cálculo de turno forestal). En el bosque natural (con ganadería y sin silvicultura) se obtuvo un valor de 165 años, presentando 213.000 ha de bosques de producción, de las cuales 150.000 ha serán de posible producción forestal neta. De esta manera surge una posibilidad anual del bosque natural de Tierra del Fuego (con la presión actual del ganado y sin silvicultura) de 913 ha anuales. Este valor se encuentra cercano al valor de corta anual actual, siendo ésta de entre 800 y 1.000 ha.

\section{CUADRO 2}

Cálculo del turno promedio y de la posibilidad (ha) de los bosques productivos en Tierra del Fuego (Argentina) con presión ganadera actual y sin silvicultura.

Average rotation length and sustainable forest use (ha) in productive forests of Tierra del Fuego (Argentina) with browsing pressure and without silvicultural treatments.

\begin{tabular}{|c|c|c|c|c|c|}
\hline \multicolumn{6}{|c|}{ Bosques de producción: 213.715 ha } \\
\hline CS & $\%$ & Con ganado & Total & Con ganado & Sin ganado \\
\hline I & 1,4 & 25,0 & 2.992 & 748 & 2.244 \\
\hline II & 20,2 & 53,3 & 43.170 & 23.010 & 20.161 \\
\hline III & 31,1 & 53,2 & 66.465 & 35.360 & 31.106 \\
\hline IV & 39,2 & 53,4 & 83.776 & 44.737 & 39.040 \\
\hline V & 8,1 & 4,2 & 17.311 & 727 & 16.584 \\
\hline \multicolumn{6}{|c|}{ Superficies efectivas (con descuentos por manejo y degradación) } \\
\hline CS & Con ganado & Sin ganado & & & \\
\hline I & 411 & 1.907 ha & & & \\
\hline II & 12.655 & 17.137 ha & & & \\
\hline III & 19.448 & 26.440 ha & & & \\
\hline IV & 24.605 & 33.184 ha & & & \\
\hline V & 400 & 14.096 ha & & & \\
\hline \multicolumn{6}{|c|}{ Superficie total efectiva: 150.283 ha } \\
\hline \multicolumn{6}{|c|}{ Ponderación de los datos } \\
\hline & \multicolumn{2}{|c|}{ Turno (años) } & & & \\
\hline CS & Con ganado & Sin ganado & & & \\
\hline I & 127 & 119 & & & \\
\hline II & 147 & 135 & & & \\
\hline III & 167 & 153 & & & \\
\hline IV & 190 & 172 & & & \\
\hline V & 200 & 180 & & & \\
\hline \multicolumn{6}{|c|}{ Turno promedio ponderado: 165 años } \\
\hline \multicolumn{6}{|c|}{$\begin{array}{l}\text { Posibilidad anual de los bosques de Tierra del Fuego (Argentina) } \\
\qquad 913 \text { h/año (con ganado y sin silvicultura) }\end{array}$} \\
\hline
\end{tabular}


BOSQUE 25(1): 29-42, 2004

Turno de corta y posibilidad de los bosques de lenga (Nothofagus pumilio) en Tierra del Fuego (Argentina)

b) Considerando el aprovechamiento de los bosques sin ganadería. La forma más simple de mejorar la posibilidad de los bosques de producción, es eliminando alguno de los factores que limitan o influyen sobre la duración del turno forestal. La ganadería es el más importante de todos por su influencia directa sobre el crecimiento y establecimiento de los renovales. Por otra parte, la ley forestal provincial $\mathrm{N}^{\circ} 145$ de Tierra del Fuego (Argentina) prohíbe el pastoreo del ganado en el bosque en regeneración, pero que no se respeta en la práctica. De realizarse un Plan de Uso de la Tierra, y de eliminarse la presión de la ganadería en el bosque de producción, los valores antes enumerados se modificarían positiva y significativamente (cuadro 3). El turno forestal promedio disminuiría a 159 años, siendo algo menor. En los bosques de producción las superficies efectivas aumentarían (por no degradarse el bosque) a 181.000 ha, quedando una posibilidad anual de 1.146 ha. Este valor está por encima de los límites de las tasas de corta actuales en Tierra del Fuego.

\section{CUADRO 3}

Cálculo del turno promedio y de la posibilidad (ha) de los bosques productivos en Tierra del Fuego (Argentina) sin presión ganadera y sin silvicultura.

Average rotation length and sustainable forest use (ha) in productive forests of Tierra del Fuego (Argentina) without browsing pressure and without silvicultural treatments.

\begin{tabular}{|c|c|c|}
\hline \multicolumn{3}{|c|}{ Bosques de producción: 213.715 ha } \\
\hline \multirow[b]{2}{*}{$\mathrm{CS}$} & \multirow[b]{2}{*}{$\%$} & \multirow{2}{*}{$\frac{\text { Superficie (ha) }}{\text { Total }}$} \\
\hline & & \\
\hline I & 1,4 & 2.992 \\
\hline II & 20,2 & 43.170 \\
\hline III & 31,1 & 66.465 \\
\hline IV & 39,2 & 83.776 \\
\hline $\mathrm{V}$ & 8,1 & 17.311 \\
\hline \multicolumn{3}{|c|}{ Superficies efectivas (con descuentos por manejo) } \\
\hline CS & \multicolumn{2}{|l|}{ Sin ganado } \\
\hline I & \multicolumn{2}{|l|}{2.543 ha } \\
\hline II & \multicolumn{2}{|l|}{36.695 ha } \\
\hline III & \multicolumn{2}{|l|}{56.496 ha } \\
\hline IV & \multicolumn{2}{|l|}{71.210 ha } \\
\hline $\mathrm{V}$ & \multicolumn{2}{|l|}{14.714 ha } \\
\hline \multicolumn{3}{|c|}{ Superficie total efectiva: 181.658 ha } \\
\hline \multicolumn{3}{|c|}{ Ponderación de los datos } \\
\hline & \multicolumn{2}{|l|}{ Turno (años) } \\
\hline CS & \multicolumn{2}{|l|}{ Sin ganado } \\
\hline I & \multicolumn{2}{|l|}{119} \\
\hline II & \multicolumn{2}{|l|}{135} \\
\hline III & \multicolumn{2}{|l|}{153} \\
\hline IV & \multicolumn{2}{|l|}{172} \\
\hline $\mathrm{V}$ & \multicolumn{2}{|l|}{180} \\
\hline \multicolumn{3}{|c|}{ Turno promedio ponderado: 159 años } \\
\hline \multicolumn{3}{|c|}{$\begin{array}{l}\text { Posibilidad anual de los bosques de Tierra del Fuego (Argentina) } \\
\qquad 1.446 \mathrm{~h} / \text { año (sin ganado y sin silvicultura) }\end{array}$} \\
\hline
\end{tabular}


c) Considerando la aplicación de tratamientos silvícolas. La silvicultura es una herramienta que se utiliza para manejar un bosque y poder alcanzar los objetivos de producción propuestos. En este caso los objetivos planteados para el manejo forestal de este trabajo fueron: (a) mantener un sistema regular sustentable y (b) obtener la máxima renta de madera y productos posibles a lo largo del turno forestal. A través de este criterio se planteó un manejo silvícola que utiliza como tratamiento de regeneración a un sistema de cortas de protección y como tratamiento intermedio a raleos fuertes por lo bajo. Por otra parte, un bosque manejado mejora las características propias de producción (volumen, sanidad, forma y crecimiento) (6), que incrementaría aún más la posibilidad de los bosques (en tiempo y producto). No es un gasto lo que se destina a la silvicultura; es, por el contrario, una inversión para el futuro. Por siglos, los bosques de Tierra del Fuego se mantuvieron dentro de una dinámica natural que culmina con una enorme acumulación de madera con un alto valor intrínseco. Hoy se aprovechan casi exclusivamente estos bosques primarios, obteniendo grandes volúmenes de aprovechamiento y no se invierte nada para acelerar o asegurar su repoblación, dejando la producción del rodal a futuro bajo una dinámica natural. Lo antes expuesto también ocurrió en los países desarrollados (por ejemplo, en Estados Unidos) a fines del siglo XIX y principios del siglo XX (podemos comparar nuestra coyuntura con la de aquella época forestal). En los países desarrollados actualmente se considera a los bosques primarios como de altísimo valor comercial respecto de los bosques de segundo crecimiento originados a partir de plantaciones. Sin embargo, la mayor ventaja de la silvicultura es poder aumentar la posibilidad del bosque, de modo de poder satisfacer la demanda actual de madera para las industrias forestales, y poder aumentar aún más el volumen de productos del bosque para poder cubrir el crecimiento de las industrias instaladas, y a través de ello crear empleo para el sector forestal. La implementación de inversiones en silvicultura (por préstamos, subsidios o manejo fiscal) podría asegurar que el manejo del recurso sea sostenible. Suponiendo que el bosque se maneje dentro de una propuesta silvícola (que elimina a la ganadería como alternativa válida de combinación productiva) el turno forestal promedio ponderado disminuye a 107 años, sensiblemente menor que los antes planteados. La posibilidad del bosque se eleva a 1.692 ha con una superficie neta de 181.000 ha (cuadro 4). Estos valores superan ampliamente las tasas de corta actuales.

\section{CONCLUSIONES Y RECOMENDACIONES}

El turno forestal de los bosques de Nothofagus pumilio varía sensiblemente con la calidad de sitio, la presión del ganado y el manejo silvícola, siendo necesario incorporar dichas variables al realizar su estimación. No es recomendable aplicar un valor fijo para el cálculo del turno forestal, ya que se corre el riesgo de cometer grandes errores, siendo los promedios ponderados obtenidos más elevados que los sugeridos por la bibliografía en general. Las modelizaciones realizadas presentan una metodología sencilla que puede utilizarse para obtener gran parte de los datos de base para la planificación de la posibilidad de una comarca forestal o en los predios de una empresa forestal que realice aprovechamientos a mediana o en gran escala.

En este trabajo se analizó la posibilidad de los bosques de Tierra del Fuego (Argentina). A través de los resultados se observa que la tasa de corta es similar a la posibilidad calculada. Sin embargo, no todos los bosques de producción están disponibles para ser incorporados dentro de un manejo forestal. En los últimos años se han desarrollado numerosos proyectos (reservas turísticas y reservas naturales) que no incluyen a la actividad forestal como alternativa productiva. Estos cambios en el uso de la tierra obedecen a la implementación de políticas gubernamentales que apuntan al desarrollo de otros sectores, sin una planificación a largo plazo. Cada vez que se implementa un proyecto de estas características no se tiene en cuenta la pérdida de la posibilidad en el aprovechamiento de los bosques de Tierra del Fuego (Argentina). Por otra parte, el $80 \%$ de las intervenciones se realizan en tierras fiscales, pudiendo esta tasa de corta aumentar significativamente cuando se pongan en marcha proyectos forestales privados (por ejemplo, el proyecto Río Grande en Argentina de la empresa Lenga Patagonia S.A.).

El método de aprovechamiento histórico de los bosques de Tierra del Fuego en Argentina se ha mantenido sin cambios durante las últimas décadas. Se basa en realizar nuevas aperturas de caminos y aprovechar bosques primarios, dejando aban- 
BOSQUE 25(1): 29-42, 2004

Turno de corta y posibilidad de los bosques de lenga (Nothofagus pumilio) en Tierra del Fuego (Argentina)

\section{CUADRO 4}

Cálculo del turno promedio y de la posibilidad (ha) de los bosques productivos en Tierra del Fuego (Argentina) sin presión ganadera y con silvicultura.

Average rotation length and sustainable forest use (ha) in productive forests of Tierra del Fuego

(Argentina) without browsing pressure and with silvicultural treatments.

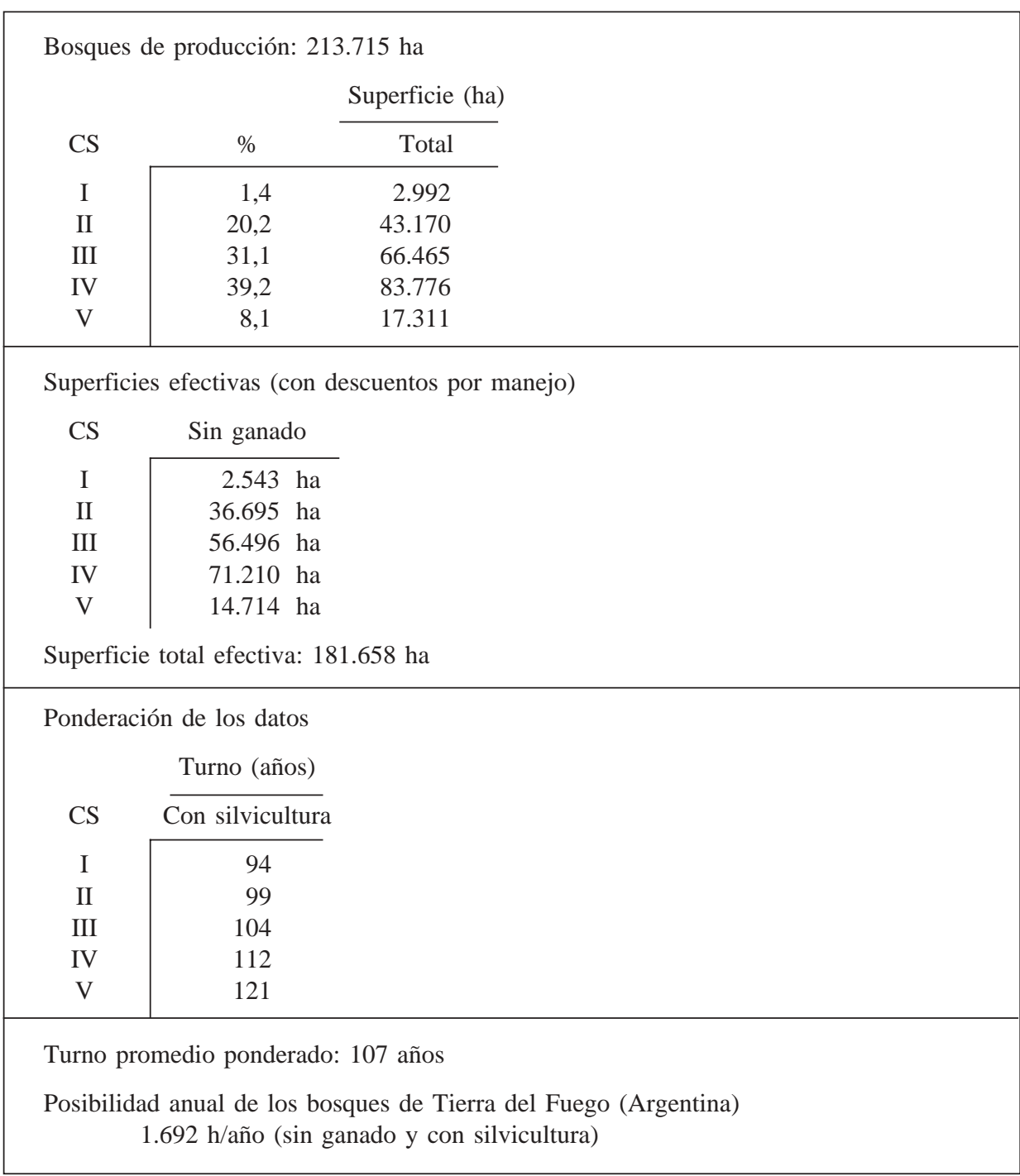

donados los bosques ya intervenidos. La planificación forestal actual se basa en el mismo criterio, siendo el $80 \%$ de las superficies de aprovechamientos sobre bosques primarios de sectores remotos. Esta falta de organización y planificación forestal ha llevado a que en varios sectores forestales ya aprovechados se instalen establecimientos que tienen otros objetivos, como ser la ganadería o el turismo. Un ejemplo de esto son los primeros cuarteles forestales estudiados y aprovechados en el sector argentino de la Isla Grande de Tierra del Fuego: Cañadón del Toro, Cañadón de la Oveja, Cañadón de Andorra, Valle de Tierra Mayor y Las Cotorras, entre otros, donde se encuentran grandes sectores en perfecto estado de regeneración y con excelentes características como para comenzar a aplicar tratamientos intermedios. Sin embargo, la mayoría de estas superficies ha sido destinada para 
otros usos no forestales, hipotecando de este modo gran parte del capital forestal para un futuro inmediato.

Si consideramos estos descuentos de superficies, se observa que en la situación actual es menor que la tasa de corta anual. Por lo que se desprende la necesidad de modificar las políticas actuales de manejo del recurso forestal. Para esto existen dos alternativas posibles:

- Modificar la tasa de corta actual, tendiendo a alcanzar los niveles de posibilidad que surjan de un estudio como el planteado en este trabajo, y modificarla año a año frente a cambios en la situación de los dominios forestales. Ante una pérdida en las superficies de bosque de producción forestal por cambios en el uso del suelo, se debe modificar tal posibilidad; por ejemplo, en Tierra del Fuego (Argentina) hay varios proyectos de reservas naturales que incorporarían e inhabilitarían el acceso a bosques de producción.

- Modificar las políticas y exigencias de manejo forestal, principalmente sobre la ganadería, sobre la aplicación de tratamientos silvícolas completos y aumentar las tasas de rendimiento del bosque. De este modo se podría alcanzar la misma posibilidad volumétrica en una superficie menor de bosque. Esto es posible aumentando los rendimientos en el bosque y en el aserradero $(11,23)$. En la actualidad se extraen en promedio $40-50 \mathrm{~m}^{3} /$ ha del bosque $(40.000$ $\mathrm{m}^{3}$ de 800-1.000 ha), pero que puede subirse fácilmente a $100 \mathrm{~m}^{3} / \mathrm{ha}$ manteniendo las calidades de productos de cosecha (7).

De no implementarse alguna de estas políticas el manejo de los bosques de Tierra del Fuego, en Argentina, no será sostenible en el tiempo, debiéndose aplicar y mantener un ciclo incompleto de producción, no pudiendo asegurar una producción estable de madera aserrada. A este problema se le suma la situación de dominio de los bosques fueguinos, cerca de la mitad de los bosques son fiscales, mientras que la otra mitad son de propiedad privada. De llevarse a cabo nuevos emprendimientos forestales en el ámbito privado, la situación actual empeoraría aún más, siendo la forma correcta de planificación donde cada dominio (el fiscal y el privado) aproveche como máximo su respectiva posibilidad.

\section{AGRADECIMIENTOS}

A la Dirección de Bosques y al Aserradero "Los Castores", y en especial al ingeniero forestal Leonardo Collado por el apoyo brindado durante la realización y discusión del presente trabajo.

\section{BIBLIOGRAFIA}

(1) RICHTER, L., J. FRANGI. Bases ecológicas para el manejo del bosque de Nothofagus pumilio de Tierra del Fuego. Revista de la Facultad de Agronomía de La Plata (Argentina), 1992, Vol. 68, p. 35-52.

(2) GROSSE W. Desarrollo de plantas de los géneros Fagus y Nothofagus en función de la luminosidad. Revisión bibliográfica. Ciencia e Investigación Forestal (Chile), 1988 , Vol. 2, No 3, p. 114-120.

(3) COZZO, D., E. MUTARELli, E. ORFILA. Plan de Investigaciones silviculturales y dasonómicas necesarias para la organización económica de los bosques subantárticos argentinos. Segundo Informe. Plan $\mathrm{N}^{\circ} 118$. Convenio Cátedra de Dasonomía - UBA y CAFPTA (Argentina), 1967. $260 \mathrm{p}$.

(4) COZZO, D., E. MUTARELli, E. ORFILA. Plan de Investigaciones silvo-dasocráticas en las etapas de ordenación, recuperación y reproducción económica de los bosques andino-patagónicos. Plan $\mathrm{N}^{\circ} 129$. Convenio Cátedra de Dasonomía - UBA y CAFPTA (Argentina), 1969. $150 \mathrm{p}$.

(5) MUTARELLI, E., E. ORFILA. Algunos resultados de las investigaciones de manejo silvicultural que se realizan en los bosques andino-patagónicos de Argentina. Revista Forestal Argentina (Argentina), 1973, Vol. 17, № 3, p. 69-75.

(6) SCHMIDT, H., A. URZUA. Transformación y manejo de los bosques de lenga en Magallanes. Ciencias Agrícolas (Chile), 1982, No 11.62 p.

(7) MARTINEZ PASTUR, G., J. CELlini, P. PERI, R. VUKASOVIC, C. FERNANDEZ. Timber production of Nothofagus pumilio forests by a shelterwood system in Tierra del Fuego (Argentina). Journal of Forest Ecology and Management (Holanda), 2000, Vol. 134, No 1-3, p. 153-162.

(8) MARTINEZ PASTUR, G., J. M. CELLINI, M. V. LENCINAS, R. VUKASOVIC, R. VICENTE, F. BERTOLAMI, J. GIUNCHI. Modificación del crecimiento y de la calidad de fustes en un raleo fuerte de un rodal en fase de crecimiento óptimo inicial de Nothofagus pumilio (Poepp. et Endl.) Krasser. Ecología Austral (Argentina), 2001, Vol. 11, p. 95-104.

(9) MARTINEZ PASTUR, G., C. FERNANDEZ, P. PERI Variación de parámetros estructurales y de composición del sotobosque para bosques de Nothofagus pumilio en relación a gradientes ambientales indirectos. Ciencias Forestales (Chile), 1994, Vol. 9, No 1-2, p. 11-22.

(10) MARTINEZ PASTUR, G., P. PERI, R. VUKASOVIC, S. VACCARO, V. PIRIZ CARRILLO. Site index equation for Nothofagus pumilio Patagonian forest. Phyton (Argentina), 1997, Vol. 6, $\mathrm{N}^{\mathrm{o}} 1 / 2$, p. 55-60.

(11) MARTINEZ PASTUR, G. Optimización del aprovechamiento del bosque de lenga: Análisis biométrico de las trozas y su implicancia en el aserradero - Campañas 1995-1998. Dirección de Bosques - Subsecretaría de Recursos Naturales y Ambiente Humano - Gobierno de Tierra del Fuego (Argentina), 1999, 18 de marzo. 15 p. 
(12) CALDERON, G. La explotación de Nothofagus al tapete. Chile Forestal (Chile), 1993, Vol. 11, No 1, p. 32-33.

(13) ROTHKUGEL, M. Los Bosques Andino-Patagónicos. Ministerio de Agricultura. Dirección General de Agricultura y Defensa Agrícola. Buenos Aires (Argentina), 1916, 207 p.

(14) ALFONSO, J. Los bosques de Tierra del Fuego. Revista Suelo Argentino (Argentina), 1942, Vol. 1, p. 47-51.

(15) RAEDAKE, K. Food habitats of the guanaco (Lama guanicoe) of Tierra del Fuego, Chile. Turrialba (Costa Rica), 1980, Vol. 30, p. 177-181

(16) SCHLICHTER, T. Impacto del pastoreo del ganado bovino y ovino sobre el bosque de Nothofagus spp. en Santa Cruz. Informe Técnico de INTA (Argentina), 1988, Expte $\mathrm{N}^{\circ} 1073.15 \mathrm{p}$.

(17) VEBLEN, T., M. MERMOZ, C. MARTIN, E. RAMILO. Effects of exotic deer on forest regeneration and composition in northern Patagonia. Journal of Applied Ecology (Inglaterra), 1989, Vol. 26, p. 711-724.

(18) VEBLEN, T., M. MERMOZ, C. MARTIN, T. KITZ BERGER. Ecological impacts of introduced animals in Nahuel Huapi National Park, Argentina. Conservation Biology (Holanda), 1992, Vol. 6, No 1, p. 71-83.

(19) MELLA, J. Guanacos. Informe del Subproyecto 94-14. Estudios de línea base: Proyecto Río Cóndor (Chile), 1995 $54 \mathrm{p}$.

(20) DODDS H. Efecto del ramoneo de guanacos (Lama guanicoe) sobre la regeneración de lenga (Nothofagus pumilio) en Russfin, Tierra del Fuego. Tesis de grado. Universidad de Chile (Chile), 1997. 58 p.

(21) MARTINEZ PASTUR, G., P. PERI, C. FERNANDEZ, G. STAFFIERI, D. RODRIGUEZ. Desarrollo de la regeneración a lo largo del ciclo del manejo forestal de un bosque de Nothofagus pumilio: 2. Incidencia del ramoneo de Lama guanicoe. Bosque (Chile), 1999, Vol. 20, N² p. 47-53.

(22) BONINO, N., A. SBRILLER. Comparación de las dietas del guanaco, ovino y bovino en Tierra del Fuego, Argentina. Turrialba (Costa Rica), 1991, Vol. 41, No 4, p. 452457.

(23) MARTINEZ PASTUR, G. Biometría del Inventario Forestal de la Provincia de Tierra del Fuego - Campaña 1996-1997. Dirección de Bosques - Subsecretaría de Recursos Naturales y Ambiente Humano - Gobierno de Tierra del Fuego (Argentina), 1999, 10 de marzo, 25 p. + 120 tablas.

(24) PIRIZ CARRILLO, V., S. VACCARO, G. MARTINEZ PASTUR. Informe Técnico: Funciones de volumen total y crecimiento diamétrico para bosques de segundo crecimiento de Nothofagus pumilio en Tierra del Fuego. LPPVCADIC (Argentina). 1996, 31 de enero. $13 \mathrm{p}$

(25) PERI, P., G. MARTINEZ PASTUR. Crecimiento diamétrico de Nothofagus pumilio para dos condiciones de copa en un sitio de calidad media en Santa Cruz, Argentina. Investigación Agraria: Sistemas y Recursos Forestales (España), 1996, Vol. 5, N² 2, p. 201-212.

(26) DIAZ, B., P. PERI, G. MARTINEZ PASTUR. Crecimiento diamétrico en bosques de lenga en sitios de calidad IV de Patagonia Sur. Actas Primer Congreso Latino- americano de IUFRO. Valdivia (Chile), 1998, 22-28 noviembre. Publicación en CD.

(27) MITSCHERLICH, E. Landwirtschaftliche Jahrbucher (Berlin), 1919, Vol. 53, p. 167-182.

(28) RICHARDS, F. A flexible growth functions for empirical use. Journal of Experimental Botany (Inglaterra)), 1959 , Vol. 10, No 29 , p. 290-300.

(29) ZEIDE, B. Analysis of growth equations. Forest Science (EE.UU.), 1993, Vol. 39, No 3, p. 591-616.

(30) MARTINEZ PASTUR, G., P. PERI, C. FERNANDEZ, G. STAFFIERI. Desarrollo de la regeneración a lo largo del ciclo del manejo forestal de un bosque de Nothofagus pumilio: 1. Incidencia de la cobertura y el aprovechamiento o cosecha. Bosque (Chile), 1999, Vol. 20, No 2 , p. 39-46.

(31) COLLADO, L. Los bosques de Tierra del Fuego. Análisis de su estratificación mediante imágenes satelitales para el inventario forestal de la provincia. Multequina (Argentina), 2001, Vol. 10, p. 1-16

(32) FITZGERALD, C. Plan de manejo: Cuartel Aguas Blancas, cantón b. Aserradero Guaraní SRL - Tierra del Fuego. Dirección de Bosques (Argentina), 1997. $31 \mathrm{p}$

(33) FREM, J. Plan de manejo forestal: Cuartel Cerro La Picuta. Aserradeo Isla Grande SRL - Tierra del Fuego. Dirección de Bosques (Argentina), 1998. 27 p.

(34) FERNANDEZ, C., G. MARTINEZ PASTUR, P. PERI, R. VUKASOVIC. Thinning schedules for Nothofagus pumilio forest in Patagonia, Argentina. Actas del XI Congreso Forestal Mundial. Volumen 3: D. Función productiva de los bosques. Antalya (Turquía), 1997, 13-22 octubre. Publicación en CD

(35) SCHMIDT, H., J. CALDENTEY. Tercer curso de silvicultura de los bosques de lenga. CONAF - CORMA Austral - Universidad de Chile. Punta Arenas (Chile), 1994, 12-22 enero. $95 \mathrm{p}$.

(36) SCHMIDT, H., J. CALDENTEY, S. DONOSO. Informe: Investigación sobre el manejo de la lenga, XII Región. Universidad de Chile - CONAF (Chile), 1995. 40 p.

(37) SCHMIDT, H., J. CALDENTEY, S. DONOSO, K. PEÑA. Seguimiento forestal y ambiental del uso de los bosques de lenga - XII Región. Universidad de Chile - CONAF (Chile), 1996. 37 p.

(38) WARDLE, J. The New Zealand beeches: ecology, utilisation and management. $1^{\text {ra }}$ ed. Christchurch: New Zealand Forest Service. 1984. 447 p.

(39) MARTINEZ PASTUR, G., J. CELlini, M.V LENCINAS, R. VUKASOVIC, P. PERI, S. DONOSO. Response of Nothofagus betuloides (Mirb.) Oerst. to different thinning intensities in Tierra del Fuego (Argentina). Interciencia (Venezuela), 2002, Vol. 27. $\mathrm{N}^{\mathrm{o}} 12$ p. 679-685.

(40) ZEIDE, B. Big projects, big problems. Environmental Monitoring and Assessment (EE.UU.), 1994, Vol. 33, p. 115-133.

(41) FRANKLIN, J., D. BERG, D. THORNBURGH, J. TAPPEINER. Alternative silvicultural approaches to timber harvesting: Variable retention harvest systems. In: KOHM, $\mathrm{K}$ and J. FRANKLIN. Creating a Forestry for the $21^{\text {st }}$ Century: The Science of Ecosystem Management. Washington: Island Press, 1997, p. 111-140. 